\title{
Voice Quality for Internet Protocol Based on Neural Network Model
}

\author{
Hassan Al-Wahshat ${ }^{1}$, Mohammed Al-Maitah'2 ${ }^{2}$ Takialddin Al-Smadi ${ }^{3}$ \\ ${ }^{1}$ Management Information Systems Department, Ajloun National University, Ajloun, Jordan \\ ${ }^{2}$ Computer Science Department, King Saud University, Riyadh, KSA \\ ${ }^{3}$ Department of Electronic \& Communication Engineering, Faculty of Engineering, Jerash University, Jerash, Jordan \\ Email: hasanwahchat@yahoo.com,malmaitah@ksu.edu.sa
}

How to cite this paper: Al-Wahshat, $\mathrm{H}$., Al-Maitah, M. and Al-Smadi T. (2017) Voice Quality for Internet Protocol Based on Neural Network Model. Journal of Signal and Information Processing, 8, 195-202. https://doi.org/10.4236/jsip.2017.84013

Received: September 12, 2017

Accepted: November 7, 2017

Published: November 10, 2017

Copyright $\odot 2017$ by authors and Scientific Research Publishing Inc. This work is licensed under the Creative Commons Attribution International License (CC BY 4.0).

http://creativecommons.org/licenses/by/4.0/

\begin{abstract}
The constant growth of the number of Internet users places new demands on the bandwidth of modern communication. The World Wide Web has led to the emergence of various types of traffic, Graphics information, voice data, as well as various video applications demanding their special requirements for such networks. To meet all the requests, one increase of the network is not enough. As the number of Internet users and the various networks annex offices increases with each passing day, network needs a means of control that would ensure the support of both existing and new applications and services. There is still a need to address a number of issues before it announces the VoIP as the ultimate replacement of the traditional telephone network. This work provides an overview of the major benefits of VoIP network, as well as the presentation of a new way around the problem of ensuring the high quality of service for VoIP protocol on the basis of neural network model.
\end{abstract}

\section{Keywords}

Next Generation Networks, Neural Network, VoIP, QoS, Circuit Switching, Packet Switching

\section{Introduction}

Voice over Packet switching networks and more specifically Voice over Internet Protocol) give us an alternative to the traditional circuit switching telephony networks existing today. VoIP is a strong competitor of the traditional circuit switching networks as it offers several advantages over the traditional circuit switching telephony networks. Among the advantages are:

1) Lower equipment cost, Integration of voice and data services; 
2) Lower bandwidth requirements;

3) Lower operating and management expenses. Still several issues need to be resolved before announcing VoIP as the ultimate replacement of the traditional telephone network. This paper studies survey of the main advantage of VoIP network as well as presenting a new way of going around the problem of achieving high quality of service for VoIP Traditional telephony companies using circuit switching networks for carrying voice traffic. Circuit switching builds a dedicated path from the sender to the receiver and the resources across the path are allocated to the phone call from beginning to end of the call, so circuit switching is carrying voice very well from source to destination.

VoIP technology can operate in any of the physical environment, which can be used as usual protocol IP. Such protection can be presented in the form of cable twisted pair (used in the traditional Ethernet), telephone wires, a wireless connection (IEEE 802.11).

This model-channel level-indicates that the IP protocol for the creation of frames may use different formats; it includes multi-channel (PPP multilink PPP), Frame Relay (FR) and ATM. When designing the network other options are possible, since the transmit voice also can be Ethernet, wife and other technologies of local networks. Using internet protocol (IP) as a method of voice, however, the usual IP should be supplemented by special means. In addition, scheme of labeling (marking) ie used with the task priorities (coloring), called the IP priorities, to ensure that the voice data reviewed by the system was more important for the primary transfer, than conventional data [1].

VoIP functions will be the main services in (RNG). We may have a question related to the use of VoIP: how maximize VoIP calls in low-speed networks (64 $\mathrm{kbit} / \mathrm{s}, 128 \mathrm{kbps}$ and $256 \mathrm{kbit} / \mathrm{s}$ ), while retaining some of their levels quality of service. According to the works, there are three approaches to design an IP network to maintain quality of service and achieve an optimal network. First, the use of trial and error the method is very expensive and takes time. Even this, the method cannot guarantee the achievement of an optimal network. Secondly, the thumb rule method sets certain quality of service values such as latency, jitter, and packet loss. This method can cause redundancy and inefficient network. Analytical method based on the E-model (ITU-T Recommendation G.107). Used to predict voice quality from the calculation quality of service parameters this method is invalid if changing network characteristics, the next question is: which method provides the most effective quality of service? To answer this question, you need to understand the organization of the VoIP system and choose VoIP network parameters. In addition, it is necessary to understand on the characteristics of VoIP, which affects the voice encoder, the level of packet loss, the size of the jitter buffer, and the network utility [2].

Understanding these VoIP characteristics, optimizing method used to achieve maximum VoIP calls with minimal, The IP networks used the transmission control protocol (TCP) for transmission of the packet, since it guarantees the deli- 
very of the packets at the receiver side. But for the delay sensitive voice communications, the TCP protocol is not preferred since it introduces the delay into the system by using acknowledge (ACK) scheme. For transmitting the voice packets, the user datagram protocol (UDP) is preferred in The VoIP communications voice packets are then sent over the IP network and the reverse process is carried out at the receiver side. The voice packets are and then decoded into the analog voice signals [3]

\section{Transmission Progress in VoIP}

Voice over Internet Protocol (VoIP) has become an attractive alternative of the traditional telephony networks for carrying voice for several reasons.

A Lower Equipment Cost: Circuit switching systems use proprietary hardware, proprietary operating systems, and applications running on proprietary software. This closed nature of the circuit switching systems makes an operator very careful in choosing the vendors for their systems because when they choose their vendors, they have to choose that vendor for the whole system due to the lack of interoperability between products from different vendors and this result in monolithic architecture.

Integration of Voice and Data: IP was originally designed to carry data ranging from e-mail to web browsing to ecommerce, when we combine this capability with the transport of voice, many new and advanced services can be offered. One example is a web page with a menu to allow entering the user's location, when the user presses a button to talk to a customer representative. Based on the user's location, the user will be directed to the most appropriate customer representative [4]. The VoIP communications voice packets are then sent over the IP network and the reverse process is carried out at the receiver side the voice packets are and then decoded into the analog voice signals show in Figure 1

Analog voice signal

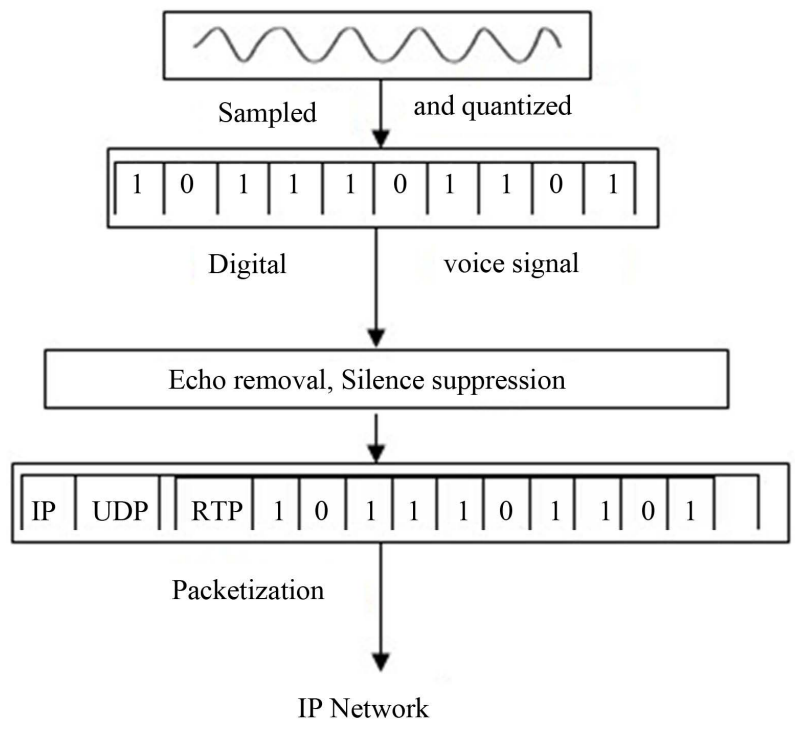

Figure 1. Voice packet preparation. 
Reduce the Bandwidth Requirements: G.711-standard for encoding voice for traditional telephony. Although the G.711 requires $64 \mathrm{kbit} / \mathrm{s}$, more efficient and modern voice coding algorithms, which allow the words must to be transferred to a different ranges from $5.3 \mathrm{kbps}$ and $32 \mathrm{kbps}$. These more advanced coding schemes can be applied to the current telephone system, but it will re-quire these encoding schemes will be carried out in virtually every telephone switchboard in the world.

Lower Operating and Management Expenses. Today enterprises need to manage two separate networks: the telephone networks, and the data (IP) networks. Implementing VoIP simplify the life for the enterprises by merging these two networks into a unified network that can carry both voice and date. This unification results in a lower operational and technical effort and at the same time leads to a reduction in expenses.

The transmission control protocol (TCP) for transmission of the packet, since it guarantees the delivery of the packets at the receiver side. But for the delay sensitive voice communications, the TCP protocol is not preferred since it introduces the delay into the system by using acknowledge (ACK) scheme. For transmitting the voice packets, the user datagram protocol (UDP) is preferred in the VoIP communications. The voice packets are then sent over the IP network and the reverse process is carried out at the receiver side. The voice packets and then decoded into the analog voice signal.

Although (VoIP) enables transport of voice traffic using the Internet Protocol (IP); however IP networks are originally designed to carry data rather than voice, therefore the most important issue in VoIP is ensuring high speech quality [5]. To ensure good voice quality, a number of issues should be addressed:

Real-time applications such as voice are extremely intolerable of delay. Mouth to Ear (M2E) delay is the delay measured from the moment a noticeable voice signal appears at the sending end (speaker's mouth) of a connection to the moment the same voice signal appears at the receiving end (listener's ear). International Telecommunication Union-Telecommunication Standardization Sector (ITU-T) Recommendation G.114 states that the M2E delay should be not more than 150 ms should be considered carefully.

Different components affect the Transmission delay of the voice signal [6].

Algorithmic Delay. Speech coders need to collect data to be able to process. The size of the data frame to be collected depends on the speech coder. Careful selection of the speech coder to be used and a balance between the voice quality with the bandwidth consumption and algorithmic delay of that speech coder should be made [7].

Processing Delay. Encoding and collecting samples for transmission purposes causes a processing delay. This delay affected by the processor execution speed and the type of the speech coding technique.

Transmission (Network) Delay. In contrast to data traffic where delay of few seconds, may be minute is rarely has an effect as soon as the data arrived correctly. 
Voice traffic is sensitive lo network delay. Specialized techniques such as resource reservation have been developed to ensure minimum network delay between sender and receiver.

Jitter Compensation Delay: Jitter buffers as discussed next are used to compensate the effect of jitter on the network. These buffers add to the overall delay [8] [9].

Packets in IP different queuing times even they both travelled the same route. If the variation of delay - also known as jitter-is keep changing, then it is difficult to adjust the delay for smooth and natural play-out of the speech signal. To minimize the effect of jitter, buffers are used to buffer the speech packets.

Packet Loss. During transmission of packets in IP networks, queues might overflow in network nodes between sender and receiver, resulting in the loss of packets. Retransmission mechanisms can be applied in the case of lost data packets to compensate the lost packets. Those retransmission mechanisms cannot be applied to real-time communications (such as voice) because the time needed to detect the Jots packets and retransmit them is Jong enough to the degree that lost packets become useless in such real-time communications (such as voice). Therefore, appropriate mechanisms are required to ensure packet Joss is minimized and if present has a minor effect. For example some complex coding techniques deal with the problem of lost packets. Techniques such as resource reservation also eliminate or reduce the packet loss over the network.

Addition to the above challenges, other requirements of the VoIP network should be presented. Among these requirements, the VoIP network should meet the five nines (99.999 percent) availability requirement described above if the VoIP is to be commercial challenge to the telephone network. To meet such condition, then redundancy of major system components should be applied and balance should be done between network cost and network quality. Other requirements such as scalability [10] [11] and applying Security techniques are also desirable and easy to add to the VoIP network.

ln VoIP we also need a specialized signaling protocol to control the start/end of the session. Determination of the coding technique to be used, issues such as: authentication to make a call, etc. We have two competing protocols in the market-the ITU-T H.323 recommendation and the Internet Engineering Task Force (IETF) SIP recommendation. These protocols and other supporting protocols such as MGCP, and MEGACO are existed in the market today. Optimizing VoIP performance is the first comprehensive, expert guide to managing, monitoring, troubleshooting, and optimizing large VoIP networks. The three leading VoIP experts combine state-of-the-art technologies to ensure that customer service level agreements are consistently met or exceeded, when a VoIP multimedia server transmits streaming traffic, audio data is periodically processed and sent over the network. The packets are then received by the user at regular intervals. During the process, the dropped packets can be discarded so as not to interrupt the playback in real time. Since the quality of voice transmission is very sensitive 
to packet loss (even a loss of $1 \%$ of packets can compromise voice quality, such packet losses caused by delay in delivery of packets can lead to deterioration in voice quality) [12]. To correctly measure the timeliness of packet arrival, it is defined as express statistical dispersion packet reception time; more formally, jitter is defined as the absolute difference between the expected time between arrivals in VoIP systems and the actual interaction time. Progress of Performance in (VoIP) is shown in Figure 2.

\section{The Need for QoS in VoIP}

Quality of Service (QoS) can be defined as a measurement of the level of service delivered to a customer. A customer that used to use the traditional telephony network is expecting something similar to what the traditional telephony is several challenges could be tackled $\mathrm{m}$ a collaborative effort to improve the QoS of the VoIP network as described in the previous section. To achieve the high quality of the traditional telephony, we should reduce the packet loss, delay, and jitter. Also, bandwidth requirements should be met.

A question may arise wondering why we should concern about improving the QoS. The answer is high-quality service is essential if an operator want to attract customers to the new IP solution for carrying voice rather than using the traditional circuit switching telephony network. No matter how low the price, if the quality of voice over IP network is not capable of competing with the traditional telephony network, the customers are not expected to be attracted to the new solution.

\section{The Proposed Solution}

Artificial Intelligent ( $\mathrm{Al}$ ) techniques are used to simulate the human intelligent

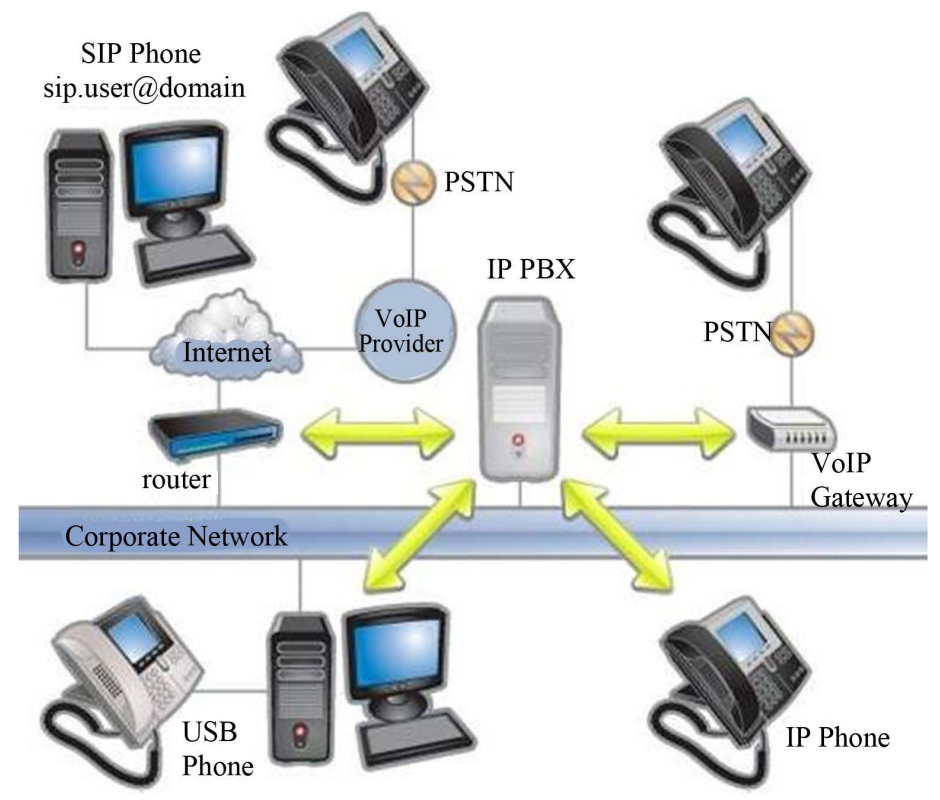

Figure 2. Progress of performance in VoIP. 
in one-way or another and they have been employed successfully in many fields [13] [14]. One of the most successful AI branches is Artificial Neural Networks (ANNs). ANNs are employed successfully in number of fields and are particularly good at pattern recognition in data.

As there are many challenges need to be tackled in VoIP such as minimizing delay, compensate packet loss, reducing the effect of jitter. These challenges will be looked into in a further detail, and will be tested under (ANNs) models. Human brain is capable lo perform such tasks as compensation of loss in the middle of speech; therefore we strongly believe that such (ANNs) techniques used as a model of human brain could be employed successfully to improve the (QoS) of VoIP.

\section{Conclusion and Future Research Directions}

VoIP is a hot research area in the world of communication. The high revenue achieved by the telecommunication companies is an attraction to develop solution that transmits voice over other media rather than the traditional circuit switching network. Several issues still need to be resolved before achieving a high quality voice over the IP networks that can compete with the traditional telephony network. These issues include such topics as: minimizing the delay, eliminating or reducing the packet loss, and reducing the effect of jitter.

\section{References}

[1] Al Smadi, T., et al. (2015) Artificial Intelligence for Speech Recognition Based on Neural Networks. Journal of Signal and Information Processing, 6, 66-72. https://doi.org/10.4236/jsip.2015.62006

[2] Larijani, H. and Radhakrishnan, K. (2010) Voice Quality in VoIP Networks Based on Random Neural Networks. 2010 Ninth International Conference on Networks (ICN), Menuires, 11-16 April 2010, 89-92. https://doi.org/10.1109/ICN.2010.23

[3] Bandung, Y., et al. (2008) Optimizing Voice over Internet Protocol (VoIP) Networks Based-On Extended E-Model. 2008 IEEE Conference on Cybernetics and Intelligent Systems, Chengdu, 21-24 September 2008, 801-805. https://doi.org/10.1109/ICCIS.2008.4670898

[4] Singh, H.P., Singh, S. and Singh, J. (2012) Performance Progress in QoS Mechanism in Voice over Internet Protocol System. International Journal of Advanced Research in Computer Science, 3, 4.

[5] Al Smadi, T. (2013) An Improved Real-Time Speech Signal in Case of Isolated Word Recognition. International Journal of Engineering Research and Applications, 3, 1748-1754.

[6] Benesty, J., Sondhi, M.M. and Huang, Y., Eds. (2007) Springer Handbook of Speech Processing. Springer Science \& Business Media, Berlin.

[7] Singh, H.P., Singh, S., et al. (2012) Performance Progress in QoS Mechanism in Voice over Internet Protocol System. International Journal of Advanced Research in Computer Science, 3, 40-44.

[8] Gubbi, J., et al. (2013) Internet of Things (IoT): A Vision, Architectural Elements, and Future Directions. Future Generation Computer Systems, 29, 1645-1660. https://doi.org/10.1016/j.future.2013.01.010 
[9] Radhakrishnan, K. and Larijani, H. (2010) A Study on QoS of VoIP Networks: A Random Neural Network (RNN) Approach. Proceedings of the 2010 Spring Simulation Multiconference, Orlando, Florida, 11-15 April 2010. https://doi.org/10.1145/1878537.1878656

[10] Khan, A., et al. (2010) Video Quality Prediction Models Based on Video Content Dynamics for H. 264 Video over UMTS Networks. International Journal of Digital Multimedia Broadcasting, 29, 1-17. https://doi.org/10.1155/2010/608138

[11] Xu, Y., Westhead, M. and Baker, F. (2004) An Investigation of Multilevel Service Provision for Voice over IP under Catastrophic Congestion. IEEE Communications Magazine, 42, 94-101. https://doi.org/10.1109/MCOM.2004.1304240

[12] Tseng, K.K., Lai, Y.C. and Lin, Y.D. (2004) Perceptual Codec and Interaction Aware Playout Algorithms and Quality Measurement for VoIP Systems. IEEE Transactions on Consumer Electronics, 50, 297-305. https://doi.org/10.1109/TCE.2004.1277877

[13] Ren, J., et al. (2010) Enhancement to E-Model on Standard Deviation of Packet Delay. 2010 3rd International Conference on Information Sciences and Interaction Sciences (ICIS), Chengdu, 23-25 June 2010, 256-259. https://doi.org/10.1109/ICICIS.2010.5534748

[14] Schatz, R., et al. (2013) From Packets to People: Quality of Experience as a New Measurement Challenge. In: Biersack, E., Callegari, C. and Matijasevic, M., Eds., Data Traffic Monitoring and Analysis, Springer Berlin Heidelberg, 219-263. https://doi.org/10.1007/978-3-642-36784-7_10 